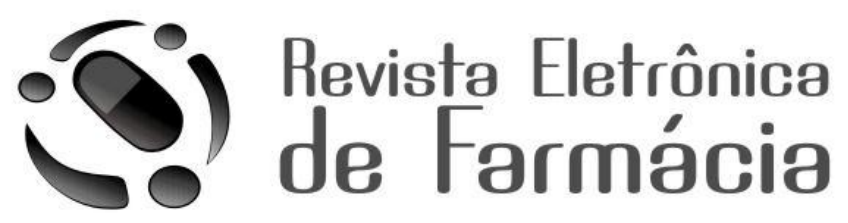

REF - ISSN 1808-0804 Vol. IX (3), 43 - 58, 2012.

\title{
ESTUDO DESCRITIVO E COMPARATIVO DE PROCEDIMENTOS EM FARMACOVIGILÂNCIA NO BRASIL.
}

\author{
DESCRIPTIVE AND COMPARATIVE STUDY OF PROCEDURES IN \\ PHARMACOVIGILANCE, BRAZIL.
}

ESTUDIO DESCRIPTIVO Y COMPARATIVO DE LOS PROCEDIMIENTOS EN DROGAS EN BRASIL.

\author{
Luciano Carlos Vieira de Mariz ${ }^{1 *}$, Fabiana Gatti de Menezes ${ }^{2}$. \\ ${ }^{1}$ Graduado em Farmácia e Bioquímica pela Universidade Nove de Julho. \\ ${ }^{2}$ Gerente de Economia da Saúde e desfecho, Abbott Laboratórios, Docente do curso de \\ pós-graduação em Farmácia Oncológica do Hospital A.C. Camargo. \\ *autor para correspondência: luciano.mariz@uninove.edu.br
}

Recebido em 26/05/2012, Aceito em 27/12/2012

\begin{abstract}
RESUMO
O uso de medicamentos causa impactos na sociedade através de benefícios sociais e econômicos ou pelo o aumento dos custos que envolvem a atenção à saúde. A coleta de dados entre os anos de 2000 e 2009 sobre os procedimentos da Agência Nacional de Vigilância Sanitária (ANVISA) quanto ao controle da comercialização de produtos farmacêuticos foi realizada a partir da consulta ao site da ANVISA (www.anvisa.gov.br) e do Food and Drugs Administration (FDA) (www.fda.gov). O ano de 2006 foi o que mais gerou alertas nacionais chegando ao total de 20 alertas e por outro lado o FDA teve o
\end{abstract}


de Mariz, L.C.V.; de Menezes, F. G. Revista Eletrônica de Farmácia Vol. IX (4), 43 - 58, 2012.

ano de 2008 com 100 alertas gerados. O Brasil vem aumentando a quantidade de alertas de farmacovigilância, porém ainda está longe dos índices do FDA.

Palavras chaves: Farmacovigilância, Reações adversas a Medicamentos, Interação Medicamentosa.

\section{ABSTRACT}

The medication use cause impacts on society through social and economic benefits or the increased costs to health care. The data collection between the years 2000 and 2009 on the procedures of the Agência Nacional de Vigilância Sanitária (ANVISA) in controlling the marketing of pharmaceutical products was carried out from consulting the Web site ANVISA (www.anvisa.gov.br) and the Food and Drug Administration (FDA) (www.fda.gov). The year was 2006 which generated more alerts coming to the national total of 20 warnings and then the FDA took the year 2008 with 100 alerts generated. Brazil has been increasing the amount of pharmacovigilance alerts, but is still inadequate compared with FDA.

Keywords: Pharmacovigilance, Adverse Drug Reactions, Drug Interactions.

\section{INTRODUÇÃO}

O uso de medicamentos causa impactos na sociedade através de benefícios sociais e econômicos ou pelo aumento dos custos que envolvem a atenção à saúde no caso de uso inadequado, ocasionando reações adversas ao medicamento (RAM), ou até mesmo óbito.

A Organização Mundial de Saúde (OMS) conceitua RAM como qualquer efeito nocivo e não intencional que ocorreu na vigência do uso do medicamento, que dever ser usado como tratamentos profiláticos, terapêuticos ou em diagnósticos sempre sendo utilizados em doses recomendadas ${ }^{(1,2)}$.

As RAMs vêem sendo classificadas por distintos autores, sendo que uma das classificações amplamente utilizada é a discutida por Rawlins e Thompson, que agrupam as reações como do tipo $A$ ou previsíveis e do tipo $B$ ou imprevisíveis ${ }^{(1,2-5)}$. As reações do tipo $A$, relacionadas à atividade farmacológica da droga, ou seja, sua cinética e dinâmica que podem provocar um efeito exagerado (1-3). Alguns exemplos de reações causadas pelo uso de medicamentos são: hipoglicemia com antidiabéticos, hemorragias causadas por anticoagulantes, nefrotoxicidade por uso de aminoglicosídeos, bradicardia pelo uso de bloqueadores $\beta$ adrenérgicos, entre várias outras reações. Geralmente estes tipos de efeitos indesejáveis estão diretamente relacionados com a variabilidade interindividual, ou seja, cada organismo responde de maneira diferente ao uso de determinadas drogas. Nestes casos é avaliado o ajuste da dose ou até mesmo 
de Mariz, L.C.V.; de Menezes, F. G. Revista Eletrônica de Farmácia Vol. IX (4), 43 - 58, 2012.

a troca por outro fármaco, as RAMs do tipo A são responsáveis por aproximadamente $80 \%$ dos casos de efeitos adversos causados pelo uso de medicamentos em doses terapêuticas, porém sua taxa de mortalidade é alta de sua letalidade baixa ${ }^{(1,3,4)}$. Já as reações do tipo B (bizarras ou imprevisíveis), não estão diretamente relacionadas à atividade farmacológica do medicamento, este tipo de reação tem uma ligação com a susceptibilidade individual de cada paciente do que com os efeitos intrínsecos do fármaco, o efeito ocorre independentemente da dose administrada e são imprevisíveis seguidos de uma alta mortalidade, muitos desses efeitos não são detectados nos estudos durante a fase de pesquisa clínica devido sua baixa incidência ${ }^{(2-4,6)}$. As reações do tipo B são produzidas por alterações na formulação farmacêutica, como decomposição de substâncias ativas além de seus excipientes, hipersensibilidade, idiossincrasia e intolerância (1-7). Diferentemente das reações do tipo $A$, apresentam incidência de mortalidade baixa, porém a letalidade pode ser muito alta $(2,4,7)$. O tratamento consiste na suspensão do fármaco, além de um acompanhamento clínico do paciente, as reações do tipo B são responsáveis por cerca de 6 a $10 \%$ das RAMs $^{(1,3)}$.

Outros autores, como Wills e Brown, classificaram as reações que não se enquadravam como as do tipo $A$ e $B$, e com isso incluíram tipos C, D, E e F. As reações do tipo C: estão relacionadas com os efeitos resultantes do uso crônico de medicamentos; do tipo D: efeito retardado do medicamento; do tipo $\mathrm{E}$ : efeito de retirada do medicamento, melhor conhecido como abstinência e as do tipo $\mathrm{F}$ estão relacionados com a falha terapêutica do fármaco ${ }^{(1,2,6,8)}$.

As RAMs podem também ser classificadas de acordo com sua intensidade no organismo, gravidade ou de acordo com sua relação casual em: leve, moderada, grave ou letal ${ }^{(1-3,6,7)}$.

A ANVISA não oferece a devida atenção aos problemas relacionados aos medicamentos como reações adversas e interações medicamentosas, sendo que sua maior preocupação recai sobre as boas práticas de fabricação e falta de registros das empresas. Assim, é importante estabelecer parâmetros que avaliem a situação brasileira com o encontrado internacionalmente.

Este trabalho teve como objetivo verificar os motivos dos alertas de farmacovigilância gerados pela ANVISA e compará-los com os alertas gerados pelo FDA, bem como orientar a população sobre os problemas relacionados aos medicamentos quando usado inapropriadamente.

\section{MATERIAS E MÉTODOS}

A coleta de dados sobre os procedimentos da ANVISA quanto ao controle da comercialização de produtos farmacêuticos foi realizada a partir da 
de Mariz, L.C.V.; de Menezes, F. G. Revista Eletrônica de Farmácia Vol. IX (4), 43 - 58, 2012.

consulta ao site da ANVISA

frequência de eventos, sendo o número (www.anvisa.gov.br) e do FDA total de 626 fármacos entre suspensão e (www.fda.gov) na área referente à Farmacovigilância. Foram coletados dados a partir do ano de 2000 até o ano de 2.009 dos medicamentos, suas associações e fitoterápicos. Trata-se de um estudo descritivo sobre a identificação dos produtos farmacêuticos que são retirados do mercado, qual o motivo da retirada. Também serão descritos e avaliados os alertas nacionais e internacionais e as consequências dos mesmos quanto à retirada de um produto farmacêutico do mercado. Os dados serão apresentados na forma de interdição, fitoterápicos 25, 67 alertas nacionais e 543 internacionais sendo um total de 520 fármacos que geraram alertas e 561 substâncias ativas.

\section{RESULTADOS}

A partir da coleta de dados sobre os fármacos suspensos conforme tabela 1 , podemos perceber que as classes terapêuticas com maior porcentagem de suspensão foram: antibióticos, seguidos por antineoplásicos e antihipertensivos.

Tabela 1. Porcentagem dos fármacos recolhidos entre os anos de 2001 a 2009, a partir de consulta no site da ANVISA, devido suspensão de uso.

\begin{tabular}{ccccc}
\hline Antibióticos & Antineoplásicos & Antihipertensivos & AINES & Antiulcerosos \\
\hline $82(33)$ & $33(12)$ & $27(10)$ & $16(6)$ & $16(6)$
\end{tabular}

Número inteiro, seguido da porcentagem entre parênteses, o número total é 274 fármacos.

Quanto aos fármacos interditados, podemos observar que as classes farmacológicas com maior frequência foram: anticoagulantes, antimicóticos e antibióticos (22\%) no ano de 2000 conforme tabela 2. Em 2.001 tivemos uma maior atenção para os antihipertensivos (70,97\%); em 2.002 novamente os antihipertensivos com $(30,00 \%)$ seguidos dos antibióticos com $(20,00 \%)$, em 2005 tivemos os antitérmicos (39\%), no último ano que foi feito o levantamento em 2009 os antibióticos atingiram $(52.94 \%)$

\begin{tabular}{|c|c|c|c|c|c|c|c|c|c|c|}
\hline Classe farmacológica & 2000 & 2001 & 2002 & 2003 & 2004 & 2005 & 2006 & 2007 & 2008 & 2009 \\
\hline Anticoagulante & $2(22)$ & $1(2)$ & $1(3)$ & - & $1(3)$ & - & $2(4)$ & - & - & - \\
\hline Anti-Hipertensivo & $1(11)$ & $6(71)$ & $11(30)$ & $4(11)$ & $5(17)$ & $4(11)$ & $4(8)$ & $9(36)$ & $8(33)$ & $1(6)$ \\
\hline
\end{tabular}


de Mariz, L.C.V.; de Menezes, F. G. Revista Eletrônica de Farmácia Vol. IX (4), 43 - 58, 2012.

$\begin{array}{lcccccccccc}\text { Antimicótico } & 2(22) & 2(3) & 1(3) & - & - & - & - & - & - & 1(6) \\ \text { Antitérmico } & 1(11) & - & 3(7) & 5(14) & 2(7) & 12(39) & 8(16) & 3(13) & 4(17) & - \\ \text { Antiparasitário } & 1(11) & 5(8) & 3(7) & 4(11) & 1(3) & 1(4) & 2(4) & - & 1(4) & - \\ \text { Antibiótico } & 2(22) & - & 7(20) & 4(11) & 8(24) & 4(14) & 12(24) & 7(27) & 3(13) & 8(53) \\ \text { AIES } & - & 2(3) & - & 4(11) & 1(3) & 2(7) & 2(4) & - & - & - \\ \text { Anticonvulsivo } & - & 1(2) & 2(5) & - & - & - & 2(4) & - & - & - \\ \text { Antiulceroso } & - & 3(5) & - & 4(11) & 1(3) & 1(4) & 1(2) & 4(14) & 1(4) & - \\ \text { Antianêmico } & - & 1(2) & - & 1(4) & 1(3) & - & 3(6) & - & - & - \\ \text { Antiviral } & - & - & 1(3) & 1(4) & - & - & 2(4) & - & - & - \\ \text { AINES } & - & - & 3(7) & 4(11) & 5(17) & 6(18) & 3(6) & - & 2(8) & - \\ \text { Antidiabético } & - & - & - & - & 2(7) & 1(4) & 1(2) & - & - & 1(6)\end{array}$

Cálculo da freqüência: número de interdições anual dividido pela quantidade que a classe apareceu, sendo os anos: 2000 (10), 2001 (65), 2002 (36), 2003 (36), 2004 (32), 2005 (32), 2006 (51), 2007 (25), 2008 (25) e 2009 (15) interdições.

Já os produtos fitoterápicos também apresentaram interdições por parte da ANVISA, a saber: Passiflora (3), Ginko biloba (6), Arnica (6), óleo de copaíba (6) e Tribulus terrestris (4), dados não mostrados.

Conforme tabela 3 os fármacos associados apresentaram menos interdições em comparação com os fármacos isolados, sendo a isoniazida+rifampicina, dimenidrato+piridoxina e amoxicilina+clavulanato, as associações medicamentosas com mais interdições no período de 2000 a 2009.

\begin{tabular}{lrrrrrrrrrr}
\hline $\begin{array}{l}\text { Tabela 3. Medicamentos Associados } \\
\text { Interditados }\end{array}$ & 2000 & 2001 & 2002 & 2003 & 2004 & 2005 & 2006 & 2007 & 2008 & 2009 \\
\hline Isoniazida+Rifampicina & 0 & 0 & 2 & 0 & 1 & 0 & 0 & 0 & 0 & 0 \\
Sulfametaxazol+Trimetoprima & 0 & 0 & 1 & 0 & 1 & 1 & 0 & 0 & 0 & 0 \\
Dimenidrato+Cloridrato de & & & & & & & & & & \\
Piridoxina & 0 & 0 & 0 & 2 & 0 & 0 & 0 & 0 & 0 & 0 \\
Ácido Acetilsalicilico+Cafeína & 0 & 0 & 0 & 1 & 0 & 0 & 0 & 0 & 0 & 0 \\
Amoxicilina+Clavulanato & 0 & 0 & 0 & 0 & 2 & 0 & 1 & 0 & 0 & 0 \\
Carbidopa+Levodopa & 0 & 0 & 0 & 0 & 0 & 0 & 0 & 0 & 1 & 1 \\
\hline
\end{tabular}

Fármacos associados com mais de uma interdição entre os anos de 2000 e 2009.

Entre fármacos associados que foram suspensos de comercialização conforme descritos na (tabela 4) destacam - se: isoniazida+rifampicina, 
de Mariz, L.C.V.; de Menezes, F. G. Revista Eletrônica de Farmácia Vol. IX (4), 43 - 58, 2012.

captopril+hidroclorotiazida, lidocaína+norepinefrina, com mais de uma suspensão no mesmo ano.

Tabela 4. Medicamentos Associados 200120022003200420052006200720082009 suspensos de comercialização

\begin{tabular}{llllllllll}
\hline Isoniazida+Rifampicina & 0 & 2 & 0 & 0 & 0 & 0 & 0 & 0 & 0 \\
Captopril+Hidroclorotiazida & 0 & 0 & 4 & 0 & 0 & 0 & 0 & 0 & 0 \\
Amoxicilina+Clavulanato & 0 & 0 & 0 & 0 & 1 & 1 & 1 & 0 & 0 \\
Cloridrato de Lidocaína+Bitartarato de & 0 & 0 & 0 & 0 & 0 & 2 & 0 & 0 & 0 \\
Norepinefrina & & & & & & & & &
\end{tabular}

Fármacos associados com mais de uma suspensão entre os anos de 2001 e 2009 relacionados em números inteiros.

Quanto aos alertas nacionais da ANVISA, a maioria dos alertas gerados foi de reações adversas a medicamentos $(72 \%)$, e (8\%) princípios ativos com alerta de farmacovigilância para atualização de bula, conforme figura 1 .
Os princípios ativos também apresentaram alertas para cancelamento de registro, falha terapêutica e interação medicamentosa, porém em menor proporção do que o observado para reações adversas, vide figura 1 .

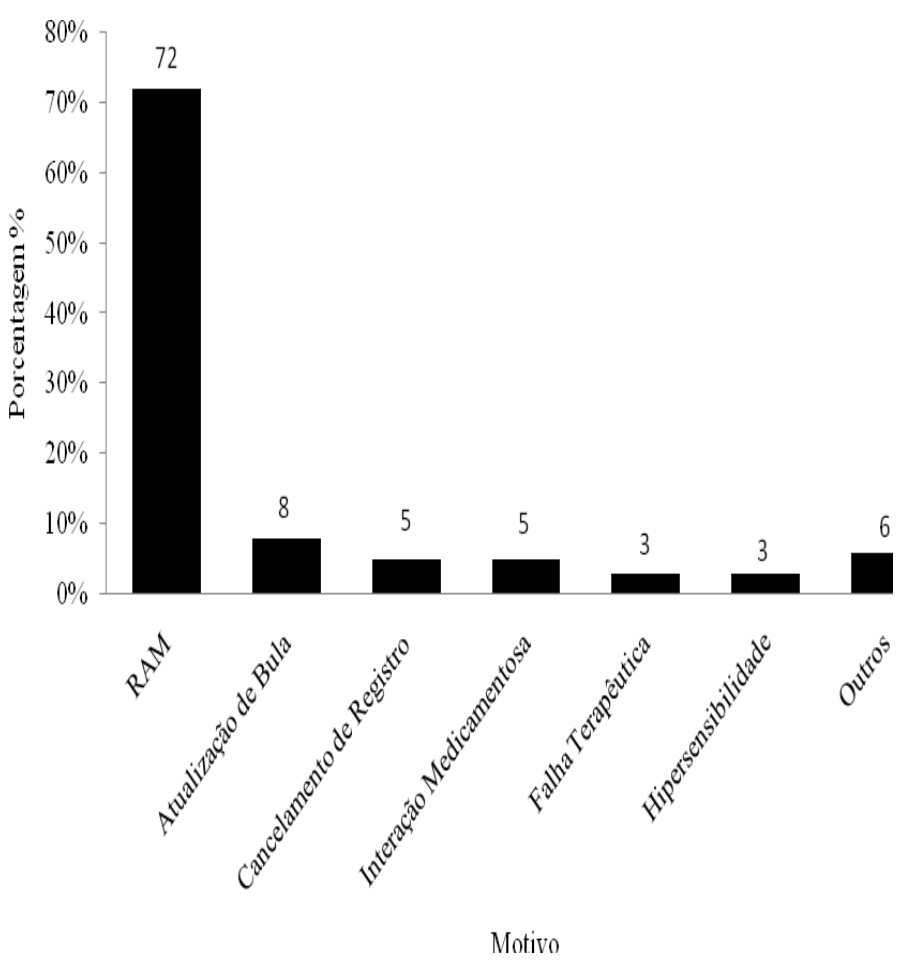

Figura 1. Motivo dos alertas nacionais de farmacovigilância, descritos em porcentagem, total de 67 alertas entre os anos de 2000 a 2009 em consulta no site da ANVISA. Outros (atualização de termos, sem registro, passou para venda com prescrição médica, suspensão de manipulação). 
de Mariz, L.C.V.; de Menezes, F. G. Revista Eletrônica de Farmácia Vol. IX (4), 43 - 58, 2012.

Na figura 2 podemos observar que dos anos, destacando-se o ano de 2006 os alertas no Brasil eram escassos, (20 alertas).

porém foram aumentando com o passar

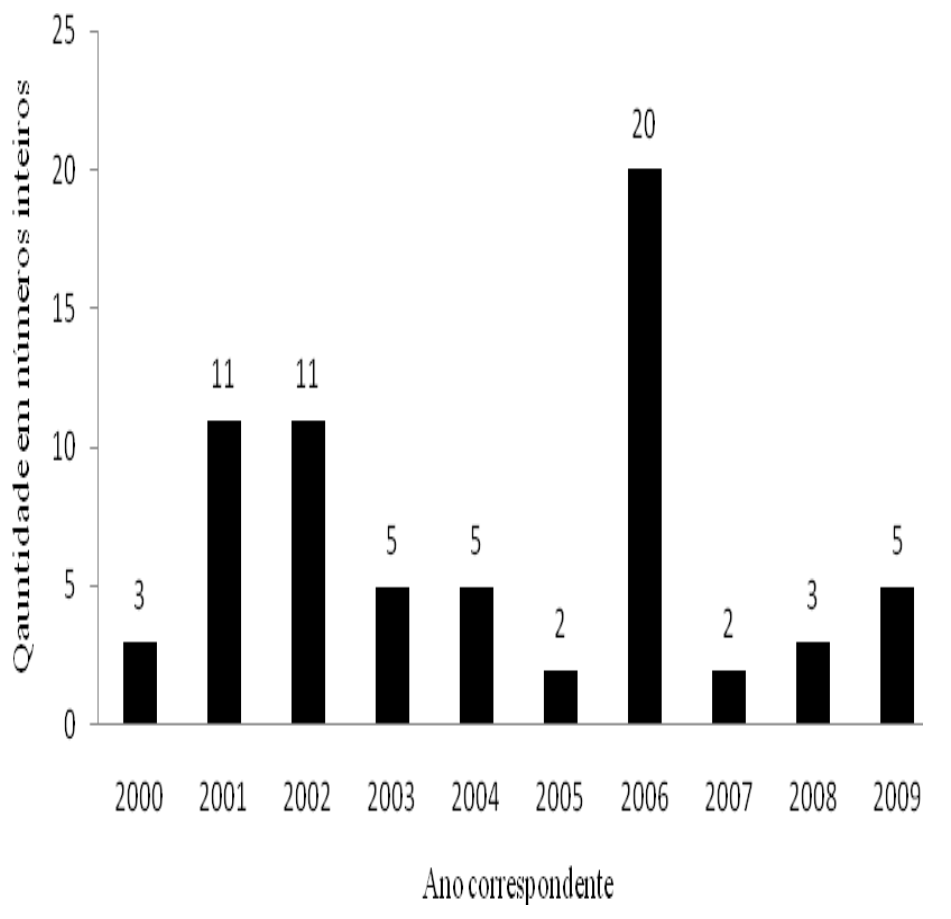

Figura 2. Alertas nacionais de farmacovigilância do ano de 2000 a 2009 em número inteiro.

Já os alertas internacionais foram mais numerosos do que os nacionais, sendo os anos mais expressivos: 2005,
2007 e 2009 conforme figura 3 totalizando 543 alertas de farmacovigilância. 
de Mariz, L.C.V.; de Menezes, F. G. Revista Eletrônica de Farmácia Vol. IX (4), 43 - 58, 2012.

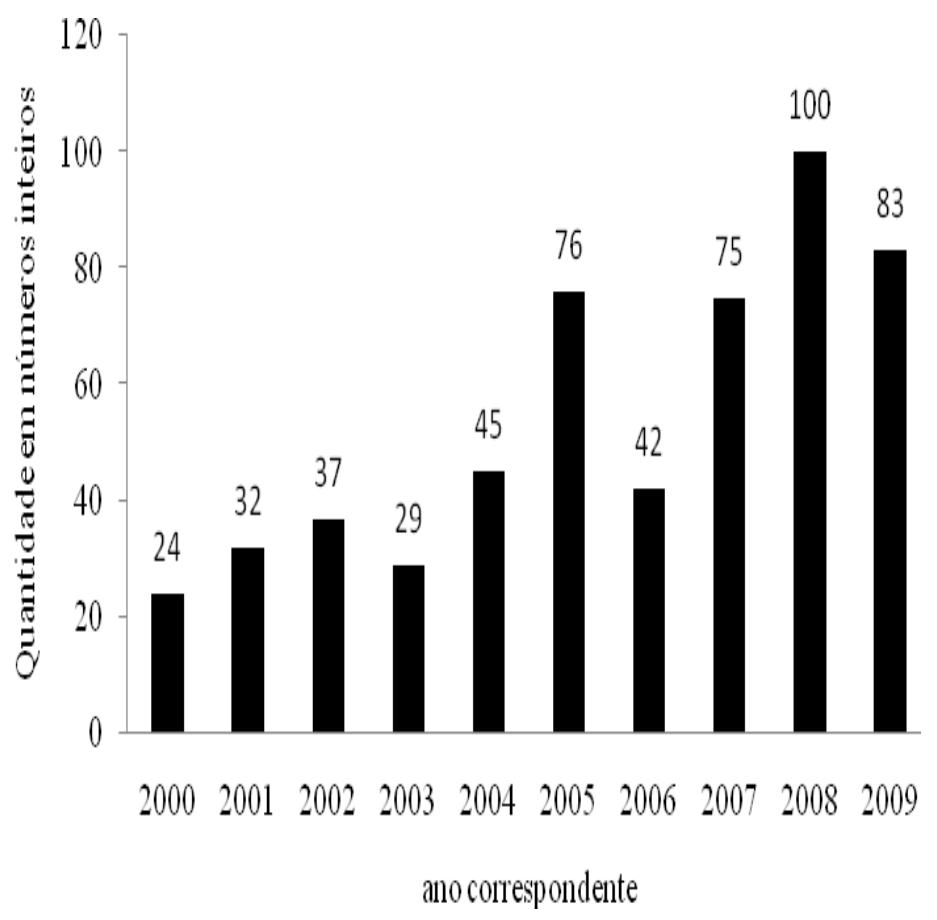

Figura 3. Quantidade de alertas internacionais gerados a cada ano em números inteiros.

Podemos observar na figura 4 que a maioria dos alertas gerados foi por motivo das RAMs $(40 \%)$ e em seguida temos os alertas de atualização de bula (23\%) e recall (14\%).

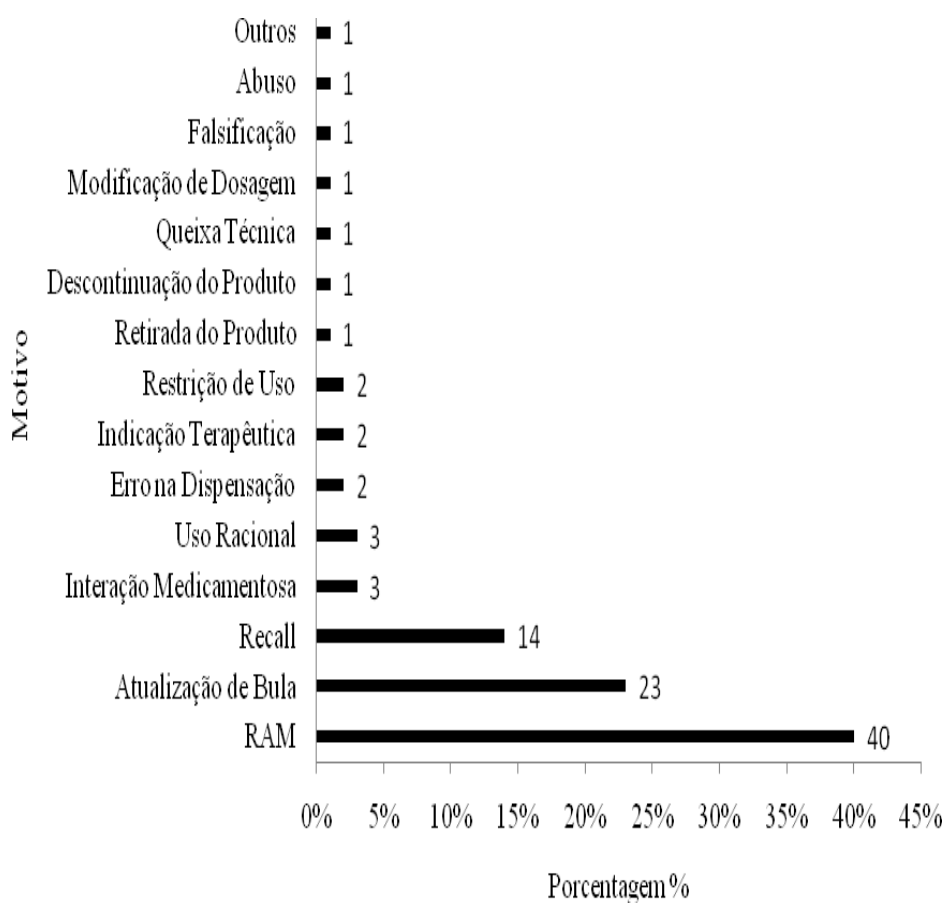

Figura 4. Principais motivos dos alertas internacionais entre os anos de 2000 e 2 009, total de 520 fármacos. Outros (nova posologia, uso proibido, novo prazo de validade, falta do medicamento). 
de Mariz, L.C.V.; de Menezes, F. G. Revista Eletrônica de Farmácia Vol. IX (4), 43 - 58, 2012.

Os fármacos que geraram os alertas internacionais, constantes na figura 5, foram os de ação no sistema nervoso (34\%), trato digestório e metabólico (14\%), os antineoplásicos e
imunomoduladores
$(13 \%)$
e
antiinfecciosos de uso sistêmico (12\%)
e, contabilizando um total de 561
substâncias ativas.

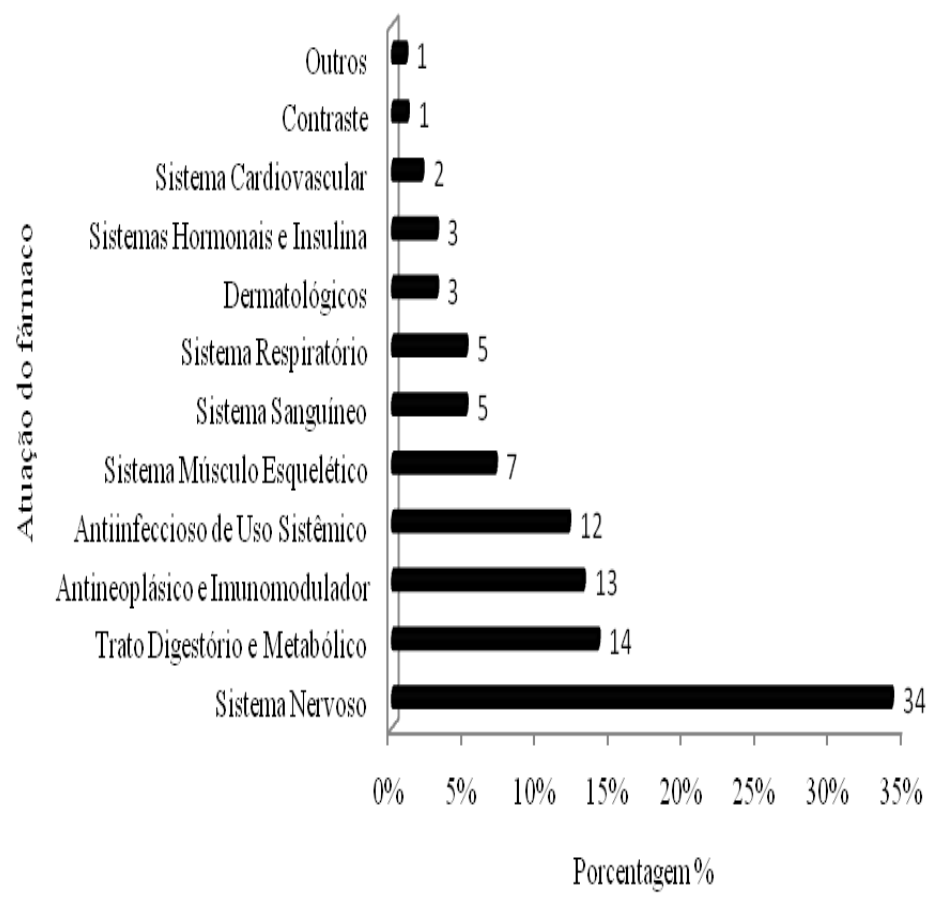

Figura 5. Principais classes de medicamentos segundo a classificação ATC preconizada pela OMS, nos anos de 2000 a 2 009, em consulta ao site do FDA quanto aos alertas internacionais. Outros (antiparasitário e órgãos sensoriais).

\section{DISCUSSÃo}

A farmacovigilância foi conceituada como a identificação e a avaliação das reações adversas principalmente as não comuns causadas pelo uso agudo e ou crônico de tratamentos farmacológicos no conjunto da população de pacientes expostos a tratamentos específicos (20). Mas, atualmente sua ação está além da segurança, sendo entendida como a ciência e as atividades relacionadas com a detecção, avaliação, compreensão e prevenção de efeitos adversos ou qualquer outro possível problema relacionado com o fármaco ${ }^{(1,20,22)}$. Seus principais objetivos: identificar reações adversas desconhecidas, quantificar o risco de reações adversas associadas ao uso específico de um determinado medicamento, identificar fatores de risco e mecanismos subjacentes a reações 
de Mariz, L.C.V.; de Menezes, F. G. Revista Eletrônica de Farmácia Vol. IX (4), 43 - 58, 2012.

adversas, informar e educar os profissionais de saúde, informar e subsidiar as autoridades sanitárias na regulamentação de medicamentos, desvios da qualidade de produtos farmacêuticos, erros de administração de medicamento, notificações de perda da eficácia, uso de medicamentos para indicações não aprovadas por base científica adequada, notificação de casos de intoxicação aguda ou crônica por produtos farmacêuticos, avaliação de mortalidade, abuso e uso errôneo de produtos, interações medicamentosas e de medicamentos com nutrientes $(4,19,20,22)$.

Colaborando para o surgimento de reações adversas temos 0 uso desenfreado de fitoterápicos e suplementos vitamínicos e minerais, considerados "produtos naturais" com a errônea idéia de que são desprovidos de efeitos nocivos. Os fitoterápicos podem interagir com outros medicamentos e os suplementos vitamínicos podem causar um quadro de hipervitaminose ${ }^{(2,13)}$. No entanto, a ausência de informações sobre toxicidade e de efeitos adversos destes produtos é o que causam o aumento do seu uso pela população, resultado da automedicação e a desinformação de pacientes e profissionais da área da saúde. Estimase que a OMS tenha recebido cerca de 5 000 relatos de efeitos indesejáveis causados por produtos fitoterápicos com mais de cem óbitos ${ }^{(13-15)}$.
A vigilância pós-cormercialização tem papel chave para a saúde pública e para o uso racional, seguro e custo efetivo de medicamento. Em países que não tem serviços reguladores estruturados, a farmacovigilância tem um papel muito importante de detectar e minimizar as consequências negativas dessa ausência de estrutura e evitar desastres relacionados com o uso de medicamentos $(1,4,7,19,20)$.

A ANVISA estabeleceu a área de Farmacovigilância em 1999 que anteriormente era integrante do setor de Segurança Sanitária de Produtos de Saúde Pós-Comercialização, e posteriormente de Medicamentos e atualmente agora faz parte do setor de Vigilância em Eventos Adversos e Queixas Técnicas. Esta área tem como responsabilidade as normas técnicas operacionais bem como a supervisão e coordenação planejar e sobre o uso seguro de medicamentos bem como a vigilância de medicamentos ${ }^{(20)}$.

Nos hospitais os antibióticos representam cerca de $20 \%$ a $50 \%$ dos medicamentos prescritos e correspondem a $50 \%$ do uso inapropriado, sendo uma das maiores causas de efeitos adversos ${ }^{(22)}$.

Outro grupo de medicamentos que deveria ser considerado com cautela são os fitoterápicos, que nos últimos anos vêm apresentando aumento de comercialização devido serem considerados uma modalidade terapêutica alternativa, e por sua 
de Mariz, L.C.V.; de Menezes, F. G. Revista Eletrônica de Farmácia Vol. IX (4), 43 - 58, 2012.

toxicidade se considerada baixa na maioria dos casos deve-se ter cuidados com sua utilização, pois já foram detectados diversos efeitos como por exemplo Kava-Kava que tem uma associação com efeitos extrapiramidais $(23,24)$. Esse aumento no consumo de fitoterápicos é notado tanto no mercado nacional quanto no internacional, a utilização desta classe de medicamentos concentra-se em pacientes do sexo feminino, que consomem: Ginkgo biloba e Passiflora ssp ${ }^{(25)}$.

A OMS define planta medicinal como sendo "todo e qualquer vegetal que possui, em um ou mais órgãos, substâncias que podem ser utilizadas com fins terapêuticos ou que sejam precursores de fármacos semi-sintéticos" (26). Grande parte da população tem consumido produtos fitoterápicos a partir do conceito errôneo de que esta classe de produtos não apresente efeitos adversos. Sabemos que podem ocorrer interações entre os próprios fitoterápicos e entre fitoterápicos e medicamentos sintéticos ${ }^{(23,24)}$.

Após a implantação da ANVISA em 1999, os alertas de farmacovigilância iniciaram de forma retraída, mas ainda é preciso aprender muito com as demais agências regulatórias (FDA, EMEA, entre outros) que geraram muito mais alertas que o órgão brasileiro em todos os aspectos, tanto em quantidade de fármacos como em quantidade de alertas anuais $^{(20)}$.
Os fármacos que tem sua atividade no sistema nervoso representam uma grande parte das prescrições brasileiras, com uma porcentagem próxima dos $14 \%$ dos 552,6 milhões de prescrições feitas, temos: antidepressivos, neurolépticos e antiepilépticos, além destes fármacos podemos citar também os anestésicos e hipnóticos. Além desses fármacos, os antibióticos se destacaram quanto à geração de alertas devido ao aumento da autoantibioticoterapia ${ }^{(27) .}$

Algumas empresas não apresentam todos os resultados de seus ensaios clínicos (positivos ou negativos) ou ainda não atualizam com frequência as respectivas bulas quanto às contraindicações, como aconteceu no caso do Refecoxibe, cujos ensaios clínicos demonstravam toxicidade cardíaca, mas mesmo assim o produto foi aprovado para venda ${ }^{(29)}$, todos os estabelecimentos de saúde deveriam ter as informações de alertas computadorizados e informações em tempo real através de um banco de dados de RAMs (30) O papel esperado pelas agências reguladoras é garantir a saúde do indivíduo, mas para isso é preciso um trabalho conjunto entre as agências reguladoras, indústrias farmacêuticas, formando um sistema transparente eficaz e robusto ${ }^{(29)}$.

É preciso que todos os profissionais de saúde trabalhem juntos para um bom diagnóstico das doenças e evitar os problemas relacionados a medicamentos $(31,32)$, mas para isso os profissionais 
de Mariz, L.C.V.; de Menezes, F. G. Revista Eletrônica de Farmácia Vol. IX (4), 43 - 58, 2012.

farmacêuticos devem prestar um serviço de farmácia clínica com todos os pacientes e trabalhar em conjuntos com os demais profissionais de saúde ${ }^{(33,34)}$. Em muitos casos devem ser evitada a politerapia o que já diminui muito os números de interações medicamentosas intoxicações ${ }^{(35,36)}$. Outra classe que deve uma grande atenção são os radiofármacos que estão tendo um crescimento considerável devido seu grande potencial de reações adversas e interações medicamentosas ${ }^{(37-39)}$.

\section{CONCLUSÃO}

Podemos concluir que o Brasil vem aumentando seus alertas de farmacovigilância, mas ainda está longe dos índices do FDA. A classe de antibióticos foi a que mais expressou suspensões no período estudado, assim como o ano de 2009 com maior quantidade de interdições. Os alertas com mais frequência nas duas agências reguladoras estudadas foi de RAM, sendo que os resultados obtidos pelo FDA foram mais significativos do que os informados pela agência reguladora brasileira. Os fármacos que geraram mais alertas internacionais foram os que atuam no Sistema Nervoso Central, e estão diretamente relacionados aos problemas relacionados aos medicamentos.
Seria importante que a ANVISA tivesse maior preocupação com os alertas e mantivesse as inspeções nas indústrias farmacêuticas que identificaram muitos princípios ativos fora das normas de BPF, principal motivo de interdições e suspensões de medicamentos. O mesmo ocorreu também com os produtos fitoterápicos, embora em menor frequência. É necessário promover uma interação maior entre a indústria farmacêutica e as agências regulatórias, para promoção da segurança dos medicamentos comercializados. Também, incentivar os estabelecimentos de saúde, principalmente farmácias, drogarias, postos de saúde e hospitais, para possibilitar a prática da atenção farmacêutica e assim promover o uso racional de medicamentos pela população. Conscientizando os profissionais de saúde quanto ao relato das reações adversas e suas responsabilidades na área de farmacovigilância. Para que isso se concretize, é preciso um trabalho em conjunto de todos os componentes envolvidos na cadeia do medicamento (indústria, agência regulatória, estabelecimentos de saúde, profissionais de saúde, pacientes) em função da segurança da profilaxia ou tratamento ao paciente.

\section{REFERÊNCIAS BIBLIOGRÁFICAS}


de Mariz, L.C.V.; de Menezes, F. G. Revista Eletrônica de Farmácia Vol. IX (4), 43 - 58, 2012.

1. Magalhães SMS, Carvalho WS. Reações adversas a medicamentos. In: Gomes MJVM, Moreira AM. Ciências Farmacêuticas uma Abordagem em Farmácia Hospitalar. São Paulo: Editora Atheneu; 2001. p.109-45.

2. Menon SZ, Lima AC, Chorilli M, Franco YO. Reações Adversas a Medicamentos. Saúde em Rev. 2005; 7(16):71-9.

3. Dias ATN, Nunes PB, Coelho HLL, Solé D. Reações alérgicas a Medicamentos. J. Pediatria. 2004; 80(4):259-66.

4. Rozenfeld S. Farmacovigilância: elementos para a discussão e perspectivas. Cad. Saúde Pública. 1998; 14(2):237-263.

5. Kawno DF, Pereira LRL, Veta JM, Freitas O. Acidentes com os medicamento como minimiza-los. Rev. Bras. Cienc. Farm. 2006; 42(4):487-88.

6. Pfaffenbach G, Carvalho OM, Mendes GB. Reações Adversas a Medicamentos como determinantes da admissão Hospitalar. Rev. Assoc. Med. Bras. 2002; 48(3):234-41.

7. Nishiyama P, Bonetti MFS, Bolm ACF, Margonato FB. Experiência de Farmacovigilância no Hospital Universitário Regional de Maringá. Acta Scientiarum. 2002; 24(3):749-50.

8. Arrais PSD. O uso irracional de Medicamentos e a Farmacovigilância no Brasil. Cad. Saúde Pública. 2002; 18(5):1478-79.

9. Hardman JG, Limbird LE, Gilman AG, editores. Goodman e Gilman As bases farmacológicas da Terapêutica. São Paulo: McGraw-Hill; 1996.

10. Wong A. Usos inadequados e os efeitos adversos de Medicamentos na prática Clínica. Jornal de Pediatria. 2005; 79(5):379-80.

11. Pereira LRL, Vecchi LUP, Baptista MEC, Carvalho D. Avaliação da Utilização de Medicamentos em pacientes idosos por meio de conceitos de Farmacoepidemiologia e Farmacovigilância. Cienc. Saúde Coletiva. 2004; 9(2):479-81. 
de Mariz, L.C.V.; de Menezes, F. G. Revista Eletrônica de Farmácia Vol. IX (4), 43 - 58, 2012.

12. Correr CJ, Pontarolo R, Ferreira LC. Riscos de problemas relacionados com medicamentos em pacientes de uma instituição geriátrica. Rev. Bras. Cienc. farm. 2007; 43(1):55-62.

13. Cruciol JMS, Thomson JC. Um estudo farmacoepidemiológico de interações medicamentosas em um hospital universitário brasileiro. Clínics. 2006; 61(6):515-20.

14. Dainesi SM. Farmacovigilância: Cada vez mais um dever de todos. Clínics. 2005; $60(4): 267-70$.

15. Brandao $M G L$, Freire N, Vianna-Soares, CD. Vigilância de fitoterápicos em Minas Gerais. Verificação da qualidade de diferentes amostras comerciais de camomila. Cad. Saúde Pública. 1998; 14(3):613-16.

16. Coelho HL, Arrais PSD, Gomes, AP. Sistema de Farmacovigilância do Ceará: um ano de experiência. Cad. Saúde Pública. 1999; 15(3):631-40.

17. Freitas MST, Romano LNS. Condições de implantação e operação da farmacovigilância na indústria farmacêutica no Estado de São Paulo, Brasil. Cad. Saúde Pública. 2007; 23(1):167-75.

18. Coelho HL. Farmacovigilância: um instrumento necessário. Cad. Saúde Pública. $1998 ; 14(4): 871-75$.

19. Rozenfeld S, Rangel ITM. A Farmacovigilância. Cad. Saúde Pública. 1998; $4(3): 336-41$.

20. Agência Nacional de Vigilância Sanitária. Área de Farmacovigilância da Anvisa. Brasília; acesso em 2007 jul 21. Disponível em: http://portal.anvisa.gov.br/wps/portal/anvisa/busca/ !ut/p/c5/04_SB8K8xLLM9MSSzPy8xBz9CP0os3hnd0cPE3MfAwMDMydnA093Uz8z00B_Aw N_Q_1wkA48Kowg8gY4gKOBvp9Hfm6qfkF2dpqjo6IiAJYj_8M!/dl3/d3/L2dJQSEvUUt3QS9Z QnZ3LzZfS1FISUcwMDI5T1Q4RDBJNFRSSDILQ09GMTE!/?WCM_GLOBAL_CONTEXT=/wps /wcm/connect/Anvisa/Anvisa/Setor+Regulado/Publicacao+Setor+Regulado/deaf7600402 11f65ae67ae54e035b7cb.

21. Castro LLC. Farmacoepidemiologia no Brasil: evolução e perspectivas. Cienc. Saúde coletiva. 1999; 4(2):405-10. 
de Mariz, L.C.V.; de Menezes, F. G. Revista Eletrônica de Farmácia Vol. IX (4), 43 - 58, 2012.

22. Andeatini R. Uso de Fitoterápicos em Psiquiatria. Rev. Bras. De Psiquiatr. 2000; 22(3):104-5.

23. Mariliére LDP, Ribeiro AQ, Brandão $M G L$, Klein $\mathrm{CH}$, Acursio FA. Utilização de Fitoterápicos por Idosos: resultados de um inquérito domiciliar em Belo Horizonte (MG), Brasil. Rev. Bras. Farmacogn. 2008; 18(Suppl 1):754-60.

24. Louro E, Lieber NSR, Ribeiro E. Eventos adversos a antibióticos em pacientes internados em umhospital universitário. Rev Saúde Pública. 2007; 41(6):1042-8.

25. Tourinho FSV, Bucaretchi F, Stephan C, Cordeiro R. Farmácias domiciliares e sua relação com a automedicação em crianças e adolescentes. J. pediatria. 2008; 84(5):41622.

26. Junior VFV, Pinto AC, Marciel MAM. Plantas medicinais: cura segura? Quím. Nova. $2005 ; 28(3): 519-528$.

27. Arrais PDS, Coelho HLL, Batista MCDS, Carvalho ML, Righi RE, Arnau JM. Perfil da Automedicação no Brasil. Rev. Saúde Pública. 1997; 31(1):71-7.

28. Fonteles MMF, Francelino EV, Santos LKX, Silva KM, Siqueira R, Viana GSB et al. Reações adversas causadas por fármacos que atuam no sistema nervoso: análise de registros de um centro de farmacovigilância do Brasil. Rev. psiquiatr. clín. 2009; 36(4):130-37.

29. Barros JAC, Martins $A$, Puente JMT, Cañás $M$, Suñé $A F$, Valsecia $M$, et $A L$. Os fármacos na atualidade. Brasília: Anvisa; 2008.

30. Zwart RJE, Uijtendaal EV, Berg MJ, Solinge WW, Egberts AC. Frequency and nature of drug-drug interation in a Dutch university hospital. $\mathrm{Br}$ J Clin Pharmacol. 2009; 68(2):187-93.

31. Fitzgerald RJ. Medication erros: the importance of an accurate drughistory. $\mathrm{Br} \mathrm{J}$ in Pharmacol. 2009; 67(6):671-5. 
de Mariz, L.C.V.; de Menezes, F. G. Revista Eletrônica de Farmácia Vol. IX (4), 43 - 58, 2012.

32. Agrawal A, Aronson JK, Britten N, Ferner RE, Fialová D, Fitzgerald RJ et al. Medication erros: problems and recommendations from a consensus meeting. $\mathrm{Br} \mathrm{J}$ Clin Pharmacol. 2009; 67(6):592-8.

33. Bond CA, Raehl CL. 2006 National Clinical Pharmacy Services Survey: Clinical Pharmacy Services, Collaborative Drug Management, Medication Erros, and Pharmacy Technology. Pharmacotherapy. 2008; 28(1):1-13.

34. Bedouch P, Baudrant M, Detavernier M, Rey C, Brudieu E, Foroni L et al. Drug supply chainsafety in hospitals: current data and experience of the Grenoble university hospital. Ann Pharm Fr. 2009; 67(1):3-15.

35. Souza PM, Neto LLS, Kusano LTE, Pereira MG. Diagnosis and controlo of polypharmacy in the elderly. Rev. Saude Publica. 2007; 41(6):1049-1053.

36. Steinman MA, Rosenthal GE, Landefeld CS, Bertenthal D, Sen S, Kaboli PJ. Conflicts and concordance between measures of medication prescribing quality. Med Care. $2007 ; 45(1): 95-9$.

37. Passarelli MC, Jacob-Filho W, Figueras A. Adverse drug reactions in an elderly hospitalised population: inappropriate prescription is a leading cause. Drugs Aging. 2005; 22(9):767-77.

38. Merle L, Laroche M L, Dantoine T, Charmes JP. Predicting and preventing adverse drug reaction in the very old. Drugs Aging. 2005; 22(5):375-92.

39. Santos OR. Radiopharmaceutical drug interations: review. Rev. Salud Publica. $2008 ; 10(3): 477-487$. 